\title{
Asylrechtliche Überlegungen zur Schutzbedürftigkeit Edward Snowdens
}

Sarah Progin-Theuerkauf*

Es gib Anhaltspunkte dafür, dass Edward Snowden die Kriterien der Flüchtlingseigenschaft nach der Genfer Flüchtlingskonvention erfüllt und er daher Asyl erhalten sollte. In jedem Fall aber müsste ihm - sofern die Gefahr besteht, dass er unter ähnlichen Bedingungen wie Chelsea Manning inhaftiert würde - aus menschenrechtlichen Aspekten (Grundsatz des Non-Refoulement) Schutz gewährt werden. In der EU steht hierfür das Instrument des subsidiären Schutzes nach der Qualifikationsrichtlinie zur Verfügung. In der Schweiz müsste Snowden, sofern er kein Asyl erhält, jedenfalls vorläufig aufgenommen werden.

I. Einleitung .23

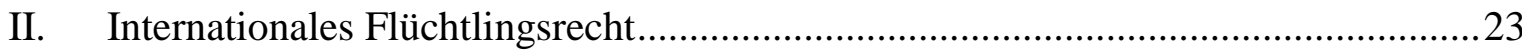

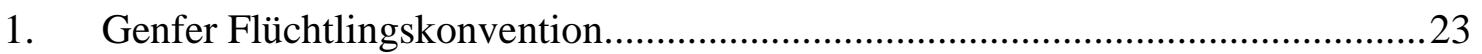

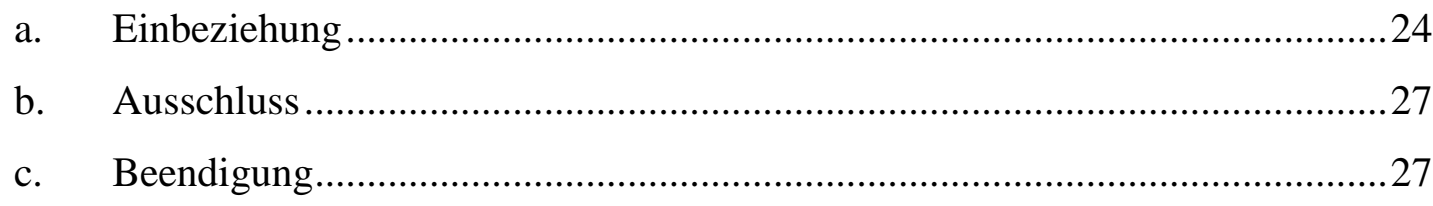

2. Europäische Menschenrechtskonvention .....................................................28

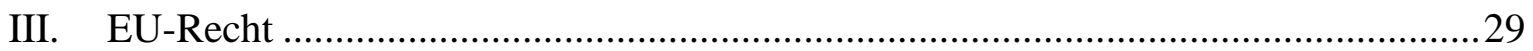

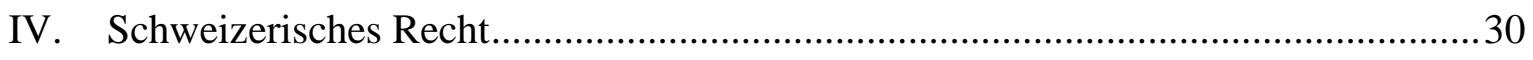

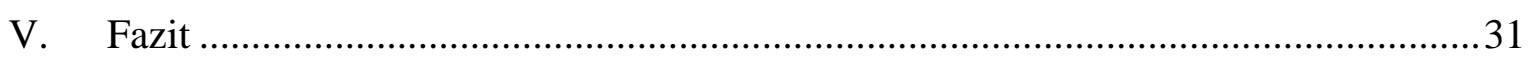

Zitiervorschlag: Sarah Progin-Theuerkauf, Asylrechtliche Überlegungen zur Schutzbedürftigkeit Edward Snowdens, in: sui-generis 2014, S. 22

URL: $\quad$ sui-generis.ch/2

DOI: $\quad$ https://doi.org/10.21257/sg.2

\footnotetext{
* Prof. Sarah Progin-Theuerkauf ist assoziierte Professorin für Europarecht und europäisches Migrationsrecht an der Universität Fribourg. Sie ist zudem Co-Direktorin des Zentrums für Migrationsrecht in Neuenburg. Themenbezogene Interessen(-bindung) der Autorin: keine.
}

Dieses Werk ist lizenziert unter einer Creative Commons Namensnennung - Weitergabe unter gleichen Bedingungen 4.0 International Lizenz. 


\section{Einleitung}

1 Im Sommer 2013 lösten die Enthüllungen des US-amerikanischen Staatsangehörigen Edward Snowden eine weltweite Spionageaffäre aus. Snowdens Informationen führten dazu, dass eine breite Öffentlichkeit Kenntnis der geheimen Überwachungsprogramme Prism (USA) und Tempora (Grossbritannien) erhielt. Der britische Guardian veröffentlichte im Juni 2013 ein entsprechendes Interview. Snowden, der im Auftrag der National Security Agency (NSA) auf Oahu (US-Bundesstaat Hawaii) als Systemadministrator für das Beratungsunternehmen Booz Allen Hamilton tätig war, flüchtete im Mai 2013 nach Hongkong, wo er seine Identität preisgab. Wenig später verliess er Hongkong wieder, sass dann aber Transitbereich des internationalen Flughafens Scheremetjewo in Moskau fest. Von dort aus stellte er Presseberichten zufolge in 21 verschiedenen Ländern, darunter auch Deutschland, Frankreich und die Schweiz, Asylgesuche. ${ }^{1}$ Auf diplomatischer Ebene wurde sein weiteres Schicksal heiss diskutiert. Im August 2013 verkündeten verschiedene Medien, Snowden habe in Russland Asyl erhalten, dies allerdings zunächst auf ein Jahr befristet und mit der Option auf Verlängerung. Nach fünf Jahren könne er dann einen Antrag auf Zuerkennung der russischen Staatsangehörigkeit stellen.

Seit Juni 2013 liegt ein Haftbefehl der USA gegen Snowden vor, u. a. wegen Spionage. Im Juli 2014 keimte die Debatte um Snowden erneut auf: Es hiess, sein Asyl laufe am
31.7.2014 aus und er müsse um Verlängerung seines Status' ersuchen. Anfang August gab sein Anwalt Anatoli Kutscherena bekannt, dass Snowden eine Aufenthaltsgenehmigung für drei weitere Jahre erteilt wurde. ${ }^{2}$ Snowden soll an einem geheimen Ort in Russland leben. ${ }^{3}$

3 Der folgende Beitrag stellt in einem ersten Schritt einige Überlegungen dazu an, ob Edward Snowden - anhand der öffentlich zugängigen Informationen $\mathrm{zu}$ seinem $\mathrm{Fall}^{4}$ durch das Internationale Flüchtlingsregime (II.) oder nach EU-Recht als schutzbedürftig einzuschätzen sein könnte (III.). In einem zweiten Schritt wird untersucht, wie seine Situation nach dem schweizerischen Asylgesetz (AsylG) zu beurteilen wäre (IV.). Da die in den Abschnitten III. und IV. anzustellenden Überlegungen weitgehend mit denen in $\mathrm{Ab}$ schnitt II. übereinstimmen, liegt der Schwerpunkt des Beitrags auf der Frage des Vorliegens der Flüchtlingseigenschaft nach der Genfer Flüchtlingskonvention.

\section{Internationales Flüchtlingsrecht}

\section{Genfer Flüchtlingskonvention}

4 Die «Magna Charta» des internationalen Flüchtlingsrechts ist das Abkommen vom 28. Juli 1951 über die Rechtsstellung der Flüchtlinge (Genfer Flüchtlingskonvention, GFK). ${ }^{5}$ Sie wird ergänzt durch das Protokoll vom 31. Januar 1967 über die Rechtsstellung der Flüchtlinge (New Yorker Protokoll). ${ }^{6}$ Das Vorliegen der Flüchtlingseigenschaft wird im

3 Handelsblatt.com vom 7. August 2014 (Russland verlängert Snowden-Asyl).

4 Insofern stützt sich der Artikel in erster Linie auf Internetquellen.

5 United Nations Treaty Series Vol. 189, 137. Vgl. auch SR 0.142.30.

6 United Nations Treaty Series Vol. 606, 267. Vgl. auch SR 0.142.301. 
Allgemeinen in drei Schritten geprüft: 1. Einbeziehung (Art. 1 A GFK), 2. Ausschluss (Art. $1 \mathrm{D}, \mathrm{E}$ und F GFK), und 3. Beendigung (Art. 1 C GFK).

Die GFK hat keine eigene Gerichtsbarkeit. ${ }^{7}$ In monistischen Systemen wie der Schweiz bilden Völkerrecht und nationales Recht aber eine einheitliche Gesamtrechtsordnung; die Normen der GFK sind innerstaatlich unmittelbar geltendes Recht und können durch nationale Gerichte unter bestimmten Umständen unmittelbar angewendet werden.

6 Die GFK enthält keine Regeln bezüglich eines allfälligen Asylverfahrens (ein solches ist somit auch nicht zwingend); die Art und Weise der Schutzgewährung bleibt dem nationalen Gesetzgeber überlassen.

7 Wichtig ist schliesslich, dass zwischen der Feststellung der Flüchtlingseigenschaft und der Asylgewährung unterschieden werden muss. ${ }^{8}$ Erstere befasst sich mit der Frage, ob eine Person unter den Anwendungsbereich der GFK (bzw. des AsylG) fällt. Die Asylgewährung hingegen ist ein staatlicher Akt, der allein in der Kompetenz des entsprechenden Staates liegt und auf dem völkerrechtlichen Grundsatz der staatlichen Souveränität beruht. In der Regel erhalten jedoch Personen Asyl, die die Kriterien der Flüchtlingseigenschaft erfüllen, sofern keine Ausschlussgründe vorliegen. Ein Staat ist aber durchaus frei, einer Person Schutz zu gewähren, die die Kriterien der Flüchtlingseigenschaft nicht erfüllt.

\section{a. Einbeziehung}

8 Die Einbeziehung richtet sich nach Art. 1 A Abs. 2 der GFK. Danach ist Flüchtling eine Person, «die, infolge von Ereignissen, die vor dem 1. Januar 1951 eingetreten sind, und aus der begründeten Furcht vor Verfolgung wegen ihrer Rasse, Religion, Nationalität, Zugehörigkeit zu einer bestimmten sozialen Gruppe oder wegen ihrer politischen Überzeugung sich ausserhalb des Landes befindet, dessen Staatsangehörigkeit sie besitzt, und den Schutz dieses Landes nicht in Anspruch nehmen kann oder wegen dieser Befürchtungen nicht in Anspruch nehmen will». Durch das New Yorker Protokoll von 1967 ist die zeitliche Beschränkung ( «Ereignisse vor dem 1. Januar 1951») sowie die sich aus Art. 1 B ergebende geographische Beschränkung ( «in Europa») weggefallen.

9 Im Folgenden soll geprüft werden, ob der USamerikanische Staatsangehörige Edward Snowden die o.g. Kriterien der Flüchtlingseigenschaft nach der Genfer Flüchtlingskonvention erfüllt. Hierfür müsste er eine begründete Furcht vor Verfolgung aus einem der fünf Verfolgungsgründe haben, sich ausserhalb seines Herkunftslandes befinden und den Schutz der USA nicht in Anspruch nehmen können oder wollen.

10 Dass er sich ausserhalb seines Heimatstaates befindet, steht bei Edward Snowden ausser Frage, da er aktuell in Russland aufhältig ist.

11 Fraglich ist allerdings die begründete Furcht vor Verfolgung aus einem bestimmten Verfol-

\footnotetext{
Einzig der Internationale Gerichtshof (IGH) könnte bei einem «Streitfall zwischen den Parteien dieses $A b$ kommens» über ihre Auslegung entscheiden (Art. 38 GFK). Eine Auslegung der Flüchtlingsdefinition ist bislang allerdings noch nie erfolgt, vgl. Francesco
}

Maiani, La définition de réfugié entre Genève, Bruxelles et Berne - différences, tensions, ressemblances, in: SFH/UNHCR (Hrsg.), Schweizer Asylrecht, EU-Standards und internationales Flüchtlingsrecht - Eine Vergleichsstudie, Bern 2009, 19, 20.

8 Caroni / Meyer / Ott, Migrationsrecht, 2. Auflage, Bern 2011, 224. 
gungsgrund. Eine allgemein gültige Definition des Begriffs Verfolgung existiert nicht. Aus Art. 33 der GFK (Prinzip des Non-Refoulement) lässt sich jedoch ableiten, dass eine Bedrohung des Lebens oder der Freiheit eines Menschen wegen eines der fünf Verfolgungsmotive stets eine Verfolgung darstellt. ${ }^{9}$

In der Regel sind Personen, die vor einer «regulären» Strafverfolgung fliehen, keine Flüchtlinge. Allerdings kann eine Bestrafung Verfolgung darstellen, wenn sie exzessiv ist (hier ist die Relation Delikt-Strafmass zu beachten) oder aus einem in der Flüchtlingsdefinition genannten Motiv erfolgt (z.B. unverhältnismässige Bestrafung wegen einer bestimmten vertretenen politischen Meinung, polit malus). Meist werden die mit dem Asylgesuch befassten Behörden die Gesetze ihres eigenen Landes als Indikator für die Beurteilung nehmen, ob eine Strafe exzessiv ist.

13 Hier könnte eine Beschränkung der Freiheit, aber auch der körperlichen Integrität bzw. der physischen und psychischen Gesundheit Snowdens drohen. Nach einer Betrachtung der Gesamtumstände gibt es zwei Ansatzpunkte für eine drohenden Verfolgung: Zum einen könnte eine Verfolgungshandlung in Form eines unfairen Prozesses, der zu einer Feststellung der Flüchtlingseigenschaft, Genf, September 1979, Ziffer 51.

10 Vgl. lemonde.fr vom 21. August 2013 (Bradley Manning condamné à trente-cinq ans de détention) und theguardian.com vom 21. August 2013 (Bradley Manning given 35-year prison term for passing files to WikiLeaks).

11 Vgl. chelseamanning.org vom 15. Juni 2014 (Chelsea Manning's appellate counsel: We'll leave no stone unturned in our quest for justice).

12 So veröffentlichten im April 2011 Bruce Ackerman (Yale Law School) und Yochai Benkler (Harvard Law School) im The New York Review of Books einen offenen Brief an Präsident Barack Obama, in dem sie die Haftbedingungen Mannings kritisierten. Vgl. nybooks.com vom 28. April 2011 (Private Manning’s exzessiven Bestrafung führt, vorliegen, zum anderen könnten auch die ihm drohenden äusserst scharfen Haftbedingungen als Verfolgung angesehen werden. Wie der Fall Chelsea (ehemals Bradley) Manning gezeigt hat, die im August 2013 wegen der Veröffentlichung verschiedener Dokumente und Videos auf WikiLeaks zu einer Freiheitsstrafe von 35 Jahren verurteilt wurde, drohen Whistleblowern in den USA äusserst harte Strafen. ${ }^{10}$ Dem Verfahren Mannings war zudem eine lange Untersuchungshaft unter extremen Bedingungen vorangegangen. Ihre Anwälte bezeichnen den Prozess als zu lang und unfair; wichtige Zeugen seien nicht gehört und der Zugang zu Dokumenten verweigert worden. ${ }^{11}$ Auch die Haftbedingungen (u.a. Isolationshaft ohne jegliche menschliche Interaktion, Verweigerung von Decken, Kopfkissen und Kleidung, mangelndes Tageslicht, etc.) von Manning wurden bereits von Juristen ${ }^{12}$, Menschenrechtsorganisationen ${ }^{13}$ und dem Sonderberichterstatter für Folter der Vereinten Nationen, Juan E. Mendez, angeprangert und sogar als Folter bezeichnet. ${ }^{14}$ Auch Abgeordnete des Europäischen Parlaments äusserten im November 2011 in einem offenen Brief an die amerikanische Regierung, den Senat und das Repräsentantenhaus Bedenken betreffend Menschenrechtsverletzungen gegenüber Manning. ${ }^{15}$

\section{Humiliation).}

13 Vgl. amnesty.de vom 24. Januar 2011 (USA: Unmenschliche Haftbedingungen für Bradley Manning). Vgl. auch focus.de vom 30. November 2012 (Keine Brille, kein ToilettenpapierWikileaks-Informant erwog in U-Haft Selbstmord) oder spiegel.de vom 11. April 2011 (Haftbedingungen von Bradley Manning: «Grausame und ungewöhnliche Bestrafung»).

14 Report of the Special Rapporteur on torture and other cruel, inhuman or degrading treatment or punishment, Addendum 4, A/HRC/19/61/Add. 4 vom 29.2.2012, 74 f., abrufbar unter http://ap.ohchr.org/documents/dpage_e.aspx?m=103.

15 Vgl. www.theguardian.com/world/2011/nov/29/ bradley-manning-mep-open-letter. 
14 Es ist nicht unwahrscheinlich, dass Snowden ein ähnliches Schicksal wie Manning droht, auch wenn er kein Angehöriger der U.S. Army war und daher - anders als Manning - nicht nach dem Uniform Code of Military Justice beurteilt würde. Insofern gelten für Snowden zwar weniger harte Regeln, ihm wird aber bislang Diebstahl von Regierungseigentum, widerrechtliche Weitergabe geheimer Informationen sowie Spionage (vgl. §§ 641, 793 (d) und 798 (a) (3) des Titels 18 des United States Code) vorgeworfen. ${ }^{16}$ Auf jedes einzelne dieser Delikte steht bereits eine Freiheitsstrafe von bis zu zehn Jahren. Auch könnte die Staatsanwaltschaft diese Strafe jeweils pro Anklagepunkt (d.h. für jedes weitergegebene Dokument) fordern; eine jahrzehntelange Haftstrafe ist daher wahrscheinlich. Insofern dürfte sich das Snowden drohende Strafmass kaum von dem unterscheiden, das Manning erhalten hat.

Als Zwischenfazit lässt sich somit festhalten, dass eine als Verfolgung zu qualifizierende Bestrafung oder Inhaftierung Snowdens nicht auszuschliessen ist.

16 Auch eine begründete Furcht vor dieser Verfolgung ist wohl anzunehmen. Hierzu ist anzumerken, dass Furcht ein subjektives Kriterium ist. Durch den Zusatz «begründet» erfährt dieses Kriterium aber eine gewisse $\mathrm{Ob}$ jektivierung. Bei der Entscheidung darüber, ob eine begründete Furcht besteht, müssen beide Kriterien berücksichtigt werde. Für die Würdigung des subjektiven Moments ist die Beurteilung der Glaubwürdigkeit des Antragstellers unerlässlich. ${ }^{17}$ Aus den gestellten Asylgesuchen ergibt sich, dass Snowden sub- jektiv - zumindest vordergründig - befürchtet, verfolgt $\mathrm{zu}$ werden. Eine weitergehende Analyse der Beweggründe Snowdens ist in diesem Rahmen nicht möglich und für die Feststellung der Begründetheit der Furcht auch nicht erforderlich. Wichtig ist aber, dass die Befürchtungen nicht unbedingt auf persönlichen Erfahrungen des Antragstellers beruhen müssen. ${ }^{18}$ Es wäre somit für den objektiven Teil zulässig und ausreichend, auf die Situation anderer Whistleblower wie die von Manning abzustellen.

17 In Bezug auf die Verfolgungsgründe der GFK muss präzisiert werden, dass die Liste der Gründe von Art. 1 A GFK abschliessend ist. In Betracht käme hier allein das Kriterium der «politischen Überzeugung». Die Tatsache, dass man eine politische Überzeugung vertritt, die von der der Regierung abweicht, ist an sich noch kein Verfolgungsgrund. Der Antragsteller muss vielmehr Ansichten vertreten, die von der Regierung nicht toleriert werden und dies muss auch den Behörden zur Kenntnis gelangt sein. ${ }^{19}$ Die betroffene Person muss gerade wegen ihrer Auffassung verfolgt werden bzw. das strafrechtliche Vorgehen gegen die Person muss politisch motiviert sein (polit malus). Es ist allerdings oftmals schwierig, hier einen Zusammenhang herzustellen, da entsprechende Massnahmen nur selten ausdrücklich mit der politischen Überzeugung begründet werden.

18 Ein polit malus dürfte hier zu bejahen sein: Snowden hatte angegeben, die entsprechenden Überwachungsprogramme aufgedeckt zu haben, weil er es nicht mit seinem Gewissen vereinbaren könne, dass die US-Regierung die Privatsphäre, die Freiheit des Internets und

Feststellung der Flüchtlingseigenschaft, Genf, September 1979, Ziffer 41.

18 Ibidem, Ziffer 43.

19 Ibidem, Ziffer 80. 
grundlegende Freiheiten weltweit mit ihrem Überwachungsapparat zerstöre. Somit handelt es sich um eine Meinungsäusserung gegen ein bestimmtes staatliches Vorgehen. Die ihm drohende empfindliche Freiheitsstrafe spricht auch dafür, dass diese Haltung nicht toleriert wird. Problematisch ist allerdings, dass Snowden Bestrafung wegen eines politischen Delikts (Spionage, widerrechtliche Weitergabe von Informationen) befürchten muss und die bei einer Verurteilung zu erwartende Strafe in Einklang mit den Gesetzen der USA stünde. Bei der Entscheidung, ob ein politischer Täter als Flüchtling anzusehen ist, müssen verschiedene Faktoren berücksichtigt werden, u.a. die Persönlichkeit des Antragstellers, seine politische Überzeugung, seine Motive und die Beschaffenheit der Rechtsordnung seines Heimatstaates. ${ }^{20}$ Unter Berücksichtigung des Ausmasses an staatlicher Überwachung, die durch Snowden aufgedeckt wurde, seiner bekundeten Überzeugung, die Bürger müssten hierüber aufgeklärt werden, ${ }^{21}$ sowie der drohenden Behandlung Snowdens durch den amerikanischen Staat im Rahmen seines Strafvollzugs (s.o.), ist nicht auszuschliessen, dass hier eine politisch motivierte Verfolgung vorliegt.

Der fehlende Schutz durch den Heimatstaat ist ebenfalls gegeben.

Als Fazit lässt sich festhalten, dass verschiedene Gründe für die Annahme sprechen, dass Edward Snowden die Flüchtlingseigenschaft nach der Genfer Flüchtlingskonvention erfüllt.

\section{b. Ausschluss}

21 Im nächsten Schritt ist zu prüfen, ob Snowden ggf. Ausschlussgründe erfüllt. Diese sind in Art. $1 \mathrm{D}$ bis $\mathrm{F}$ der GFK geregelt. Art. $1 \mathrm{D}$ nimmt Personen vom Anwendungsbereich der GFK aus, die bereits Schutz oder Beistand einer Organisation oder einer Institution der Vereinten Nationen (nicht UNHCR) geniessen. ${ }^{22}$ Der Ausschlussgrund von Art. 1 E betrifft Personen, die durch einen Staat wie eigene Staatsangehörige behandelt werden und daher nicht schutzbedürftig sind. Art. $1 \mathrm{~F}$ regelt schliesslich Fälle, in denen die betroffene Person bestimmte Handlungen begangen hat, die sie als schutzunwürdig qualifizieren (Kriegsverbrechen o.ä., schwere nichtpolitische Verbrechen ausserhalb des Aufnahmestaates, Handlungen gegen die Ziele und Grundsätze der Vereinten Nationen). ${ }^{23}$ Hier käme allein ein Ausschluss nach Art. $1 \mathrm{~F}$ wegen eines schweren nichtpolitischen Verbrechens in Betracht. Allerdings dürften die durch Snowden begangenen Delikte aus politischen Motiven begangen worden sein. Es besteht ein direkter Kausalzusammenhang zwischen den begangenen Delikten und den politischen Zielen Snowdens, die Privatsphäre und die Freiheit des Internets zu schützen. Ein Ausschlussgrund liegt damit nicht vor.

\section{c. Beendigung}

22 Anhaltspunkte für eine Beendigung der Flüchtlingseigenschaft nach Art. 1 C GFK wie der Erwerb einer neuen Staatsangehörigkeit oder die freiwillige Rückkehr in das Heimatland - liegen nicht vor.

Ibidem, Ziffer $84 \mathrm{ff}$.

21 Dieses Interesse der Bürger an einer gewissen Transparenz bei der staatlichen Überwachung und der Verhältnismässigkeit von Eingriffen ihrer Privatsphäre wäre gegen das Interesse des Staates an einem funktionierenden Geheimdienst und der Aufdeckung und Abwehr von Straftaten abzuwägen. Eine flächendeckende, systematische Aufzeichnung von Telefon- und Internetdaten aller Bürger, losgelöst von jeglichem
Verdacht auf Straftaten, lässt sich wohl nicht rechtfertigen.

22 Vgl. zur Auslegung dieser Bestimmung auch EuGH, Rs. C-31/09, Bolbol, Slg. 2010, I-5539 und Rs. C364/11, Urteil vom 1912.2012, El Kott.

23 Vgl. dazu EuGH, Rs. C-57/09 und C-101/09, B und D, Slg. 2010, I-10979. 


\section{Europäische Menschenrechtskonvention}

23 Aus Art. 3 der Europäischen Menschenrechtskonvention wird zudem das Non-Refoulement-Prinzip abgeleitet, auf dem die sog. subsidiäre bzw. komplementäre Schutzform basiert. ${ }^{24}$ Demnach darf niemand Folter oder unmenschlicher oder erniedrigender Strafe oder Behandlung unterworfen werden. Der Unterschied der genannten Formen der Misshandlung ist graduell; Folter ist dabei der schwerste Eingriff. ${ }^{25}$ Eine Definition von Folter enthält die EMRK nicht. Der Europäische Gerichtshof für Menschenrechte (EGMR) stützt sich hier auf die Begriffsbestimmung in Art. 1 der UN-Folterkonvention. ${ }^{26}$ Als unmenschliche Behandlung hat der EGMR eine Behandlung angesehen, wenn sie vorsätzlich war, ohne Unterbrechung länger andauerte und entweder eine Körperverletzung oder intensives physisches oder psychisches Leiden verursachte. ${ }^{27}$ Als erniedrigend wird eine Behandlung qualifiziert, wenn mit ihr die Absicht verbunden ist, den Betroffenen zu demütigen oder zu erniedrigen. Auch unverhältnismässig hohe Strafen, unmenschliche Verhörmethoden und besonders schlimme Haftbedingungen wie Unterbringung ohne Kleidung oder die vollständige sensorische und soziale Isolation können Art. 3 EMRK verletzen. ${ }^{28}$

24 Der Schutz des Art. 3 ist absolut ${ }^{29}$; Ausnahmen (z.B. im Fall von Terrorismus) sind nicht möglich. ${ }^{30}$

$\mathrm{Zu}$ beachten ist, dass die EMRK zwar keinen Anspruch auf Asyl bzw. Aufenthalt von Ausländern garantiert, aber eine Auslieferung oder Abschiebung für den Aufenthaltsstaat konventionsrechtlich relevant sein kann, wenn begründete Anhaltspunkte dafür vorliegen, dass die betroffene Person im Empfangsstaat einem tatsächlichen Risiko der Folter oder unmenschlicher oder erniedrigender Behandlung oder Bestrafung unterworfen ist. ${ }^{31}$ In der
24 Vgl. etwa EGMR, Urteil vom 23.2.2012, Application No. 27765/09, Hirsi Jamaa v. Italy; Urteil vom 21.1.2011, Application No. 30696/09, M.S.S. v. Belgium and Greece; Urteil vom 20.7.2010, Application No. 23505/09, N. v. Sweden; Urteil vom 17.7.2008, Application No. 25904/07, N.A. v. United Kingdom; Urteil vom 2.5.1997, Application No. 30240/96, D. v. United Kingdom. Zur subsidiären Schutzform vgl. Patricia Petermann / Christine Kaufmann, Die subsidiäre Schutzform, in: SFH/UNHCR (Hrsg.), Schweizer Asylrecht, EU-Standards und internationales Flüchtlingsrecht - Eine Vergleichsstudie, Bern 2009, 67.

25 Karpenstein / Mayer, Konvention zum Schutze der Menschenrechte und Grundfreiheiten, 2012, Art. 3, Rz. 5, 7.

26 Convention against Torture and Other Cruel, Inhuman or Degrading Treatment or Punishment; s. auch SR 0.105. Die Folterkonvention schützt - im Unterschied zur EMRK - nur bei drohender Folter, nicht aber bei unmenschlicher oder erniedrigender Behandlung vor Abschiebung.

27 Meyer-Ladewig, Europäische Menschenrechtskonvention, Handkommentar, 3. Auflage, 2011, Art. 3, Rz. 22.

28 Karpenstein / Mayer, Konvention zum Schutze der Menschenrechte und Grundfreiheiten, 2012, Art. 3, Rz. 10 ff.; Meyer-Ladewig, Europäische Menschenrechtskonvention, Handkommentar, 3. Auflage, 2011,
Art. 3, Rz. 29, 36; m.w.N.

29 Vgl. Meyer-Ladewig, Europäische Menschenrechtskonvention, Handkommentar, 3. Auflage, 2011, Art. 3, Rz. 1 ff.; Grabenwarter / Pabel, Europäische Menschenrechtskonvention, 5. Auflage, $168 \mathrm{ff}$. Anders dagegen das flüchtlingsrechtliche Non-RefoulementPrinzip in Art. 33 GFK, dessen Abs. 2 es erlaubt, einen Flüchtling aus- oder zurückzuweisen, der aus schwerwiegenden Gründen als eine Gefahr für die Sicherheit des Landes anzusehen ist, in dem er sich befindet, oder der eine Gefahr für die Allgemeinheit dieses Staates bedeutet, weil er wegen eines Verbrechens oder eines besonders schweren Vergehens rechtskräftig verurteilt wurde. Art. 33 GFK schützt zudem nur Flüchtlinge im materiellrechtlichen Sinne.

30 EGMR, Urteil vom 15.11.1996, Application No. 22414/93, Chahal v. The United Kingdom. Vgl. auch Ruedi Illes, Art. 83 AuG, Rz. 22, in : Caroni / Gächter / Thurnherr, Bundesgesetz über die Ausländerinnen und Ausländer (AuG), Bern 2010.

31 EGMR, Urteil vom 7.7.1989, Application No. 14038/88, Soering v. United Kingdom; EGMR, Urteil vom 15.11.1996, Application No. 22414/93, Chahal v. The United Kingdom. Vgl. auch Karpenstein / Mayer, Konvention zum Schutze der Menschenrechte und Grundfreiheiten, 2012, Art. 3, Rz. 24; Meyer-Ladewig, Europäische Menschenrechtskonvention, Handkommentar, 3. Auflage, 2011, Art. 3, Rz. 60; 
Übergabe des Betroffenen in die Gewalt des ausländischen Staates liegt dann eine dem ausweisenden Staat unmittelbar zurechenbare Handlung, die seine Verantwortlichkeit nach der EMRK begründet. ${ }^{32}$

Der EGMR verlangt für einen Verstoss gegen Art. 3 EMRK den Nachweis der erheblichen Wahrscheinlichkeit einer konkreten und ernsthaften Gefahr ( «real risk»). ${ }^{33}$ Die Beweislast liegt bei der betroffenen Person. ${ }^{34}$ Sofern es Edward Snowden also gelingt, schlüssig nachzuweisen, dass ihm in den USA im Rahmen seiner Haft eine unmenschliche Behandlung droht, müsste ihm jedenfalls über Art. 3 EMRK Schutz gewährt werden. Eine Auslieferung kann u.U. auch gegen Art. 6 EMRK (Recht auf ein faires Verfahren) verstossen. ${ }^{35}$

Schliesslich ist noch anzumerken, dass allfällige diplomatische Zusicherungen des Aufnahmestaates (hier der USA) den Konventionsstaat nicht von seinen Verpflichtungen nach der EMRK entbinden. ${ }^{36}$ Das tatsächliche Risiko einer Misshandlung ist stets zu prüfen. ${ }^{37}$

Mit Art. 3 EMRK nahezu deckungsgleiche Normen finden sich auch in Art. 3 der UNFolterkonvention sowie in Art. 7 des Internationalen Pakts über bürgerliche und politische Rechte (UNO-Pakt II). ${ }^{38}$

\section{EU-Recht}

29 Im EU-Recht regelt die Qualifikationsrichtlinie (QRL) von 2011 ${ }^{39}$, wer international schutzberechtigt ist. Der Begriff «internationaler Schutz» bezeichnet nach Art. 2 lit. a QRL die Flüchtlingseigenschaft und den subsidiären Schutzstatus. Die beiden Schutzformen basieren im Wesentlichen auf der Genfer Flüchtlingskonvention (Flüchtlingseigenschaft) und der durch den EGMR entwickelten Rechtsprechung zu Art. 3 EMRK (subsidiärer Schutz), wobei sich insbesondere in letzterer Hinsicht einige Abweichungen feststellen lassen (vgl. z.B. Art. 15 lit. c QRL).

30 Die Anerkennung als Flüchtling richtet sich nach Art. 9 ff. QRL. Nach Art. 9 Abs. 2 lit. c QRL kann auch eine unverhältnismäßige oder diskriminierende Strafverfolgung oder Bestrafung als Verfolgung gelten. Insofern können die oben angestellten Überlegungen auch auf das Europarecht übertragen werden.

31 Sofern man annimmt, die Kriterien der Flüchtlingseigenschaft seien nicht erfüllt, kann eine Person nach Art. 2 lit. f i.V.m. Art. 15 ff. QRL noch subsidiär schutzberechtigt sein. Art 2 lit. f QRL definiert als «Person mit Anspruch auf subsidiären Schutz» einen Drittstaatsangehörigen oder einen Staatenlosen, der die Voraussetzungen für die Anerkennung als Flüchtling
Grabenwarter / Pabel, Europäische Menschenrechtskonvention, 5. Auflage, 176; Hailbronner, Art. 3 EMRK - ein neues europäisches Konzept der Schutzgewährung?, DÖV 1999, 617, 618 f.

32 Grabenwarter / Pabel, Europäische Menschenrechtskonvention, 5. Auflage, 177.

33 Vgl. EGMR, Urteil vom 7.7.1989, Application No. 14038/88, Soering v. The United Kingdom. Danach müssen für das «real risk» begründete Tatsachen («substantial grounds») vorliegen.

34 Grabenwarter / Pabel, Europäische Menschenrechtskonvention, 5. Auflage, 180.

35 Dazu Meyer-Ladewig, Europäische Menschenrechtskonvention, Handkommentar, 3. Auflage, 2011, Art. 3, Rz. 29, 62, Art. 6, Rz. 167.
36 Karpenstein / Mayer, Konvention zum Schutze der Menschenrechte und Grundfreiheiten, 2012, Art. 3, Rz. 25.

37 Vgl. EGMR, Urteil vom 28.2.2008, Saadi v. Italy, Application No. 37201/06.

38 International Covenant on Civil and Political Rights; s. auch SR 0.103.2.

39 Richtlinie 2011/95/EU des Europäischen Parlaments und des Rates vom 13.12.2011 über die Normen für die Anerkennung von Drittstaatsangehörigen oder Staatenlosen als Personen mit Anspruch auf internationalen Schutz, für einen einheitlichen Status für Flüchtlinge oder für Personen mit Anrecht auf subsidiären Schutz und für den Inhalt des zu gewährenden Schutzes, ABl. L 337 vom 20.12.2011, 9. 
nicht erfüllt, der aber stichhaltige Gründe für die Annahme vorgebracht hat, dass er bei einer Rückkehr in sein Herkunftsland oder, bei einem Staatenlosen, in das Land seines vorherigen gewöhnlichen Aufenthalts tatsächlich Gefahr liefe, einen ernsthaften Schaden im Sinne des Art. 15 QRL zu erleiden, und auf den Art. 17 Abs. 1 und 2 QRL keine Anwendung findet und der den Schutz dieses Landes nicht in Anspruch nehmen kann oder wegen dieser Gefahr nicht in Anspruch nehmen will. Nach Art. 15 lit. b QRL gelten insbesondere Folter oder unmenschliche oder erniedrigende Behandlung oder Bestrafung eines Antragstellers im Herkunftsland als ernsthafter Schaden.

Edward Snowden müsste also zumindest als subsidiär schutzberechtigt im Sinne der EUQualifikationsrichtlinie anerkannt werden. Der Status der subsidiär Schutzberechtigten wurde mit der Neufassung der Qualifikationsrichtlinie 2011 dem Flüchtlingsstatus angeglichen, wenngleich einige Unterschiede in Bezug auf Sozialleistungen und Aufenthaltsbewilligungen bestehen bleiben. ${ }^{40}$

Möglicherweise wird auch das aktuell vor dem EuGH anhängige Vorabentscheidungsersuchen im Fall Sheperd ${ }^{41}$, in dem es um einen US-amerikanischen Deserteur geht, der in Deutschland erfolglos Asyl beantragt hat, noch einige interessante Aspekte für eine Argumentation im Fall Snowden enthalten. Mit einem Urteil des EuGH ist jedoch erst in einigen Monaten zu rechnen.

\section{Schweizerisches Recht}

34 Nach Art. 3 Abs. 1 des schweizerischen Asylgesetzes (AsylG) ${ }^{42}$ sind Flüchtlinge Personen, die in ihrem Heimatland oder im Land, in dem sie zuletzt wohnten, wegen ihrer Rasse, Religion, Nationalität, Zugehörigkeit zu einer bestimmten sozialen Gruppe oder wegen ihrer politischen Anschauung ernsthaften Nachteilen ausgesetzt sind oder begründete Furcht haben, solchen Nachteilen ausgesetzt zu werden. Art. 3 Abs. 2 AsylG definiert die ernsthaften Nachteile. Als solche gelten namentlich die Gefährdung des Leibes, des Lebens oder der Freiheit sowie Massnahmen, die einen unerträglichen psychischen Druck bewirken. Obwohl der Wortlaut von Art. 3 AsylG nicht mit dem von Art. 1 A GFK übereinstimmt, soll Art. 3 nach Auffassung des Gesetzgebers die GFK widerspiegeln. ${ }^{43}$

Bei Edward Snowden dürfte als ernsthafter Nachteil insbesondere die Kategorie der Freiheitsbeschränkungen in Frage kommen. Hier müssen die konkreten Umstände des Freiheitsentzugs berücksichtigt werden (Haftbedingungen, Beachtung der Menschenrechte durch die Sicherheitskräfte, etc.). ${ }^{44}$ Im Übrigen lassen sich die unter II. angestellten Überlegungen auch auf Art. 3 AsylG übertragen.

36 Unterschiede zwischen Schweizerischem Recht und der GFK bestehen schliesslich hinsichtlich der Ausschlussgründe bzw. bei Asylunwürdigkeit. Nach Art. 53 AsylG sind Flüchtlinge von der Asylgewährung ausgeschlossen, wenn sie wegen verwerflicher

1990, 28; Francesco Maiani, La définition de réfugié entre Genève, Bruxelles et Berne - différences, tensions, ressemblances, in: SFH/UNHCR (Hrsg.), Schweizer Asylrecht, EU-Standards und internationales Flüchtlingsrecht - Eine Vergleichsstudie, Bern 2009, 19, 22.

44 Vgl. Schweizerische Flüchtlingshilfe (Hrsg.), Handbuch zum Asyl- und Wegweisungsverfahren, Bern 2009, 171. 
Handlungen deren unwürdig sind oder die innere oder äussere Sicherheit der Schweiz verletzt haben oder gefährden. Es erfolgt lediglich ein Ausschluss vom Asyl, nicht aber von der Flüchtlingseigenschaft. Eine Person kann in diesem Fall als Flüchtling vorläufig aufgenommen werden. Sofern bereits die Flüchtlingseigenschaft verneint wird, kann auch eine vorläufige Aufnahme als Ausländer in Betracht kommen, wenn der Vollzug der Wegoder Ausweisung nicht möglich, nicht zulässig oder nicht zumutbar ist (Art. 44 AsylG i.V.m. Art. 83 AuG). Bei einer drohenden Verletzung von Art. 3 EMRK wäre der Vollzug der Wegweisung nicht zulässig (Art. 83 Abs. $3 \mathrm{AuG}){ }^{45}$

Hier wäre zu überlegen, ob bei einer Aufnahme Snowdens die äussere Sicherheit der Schweiz gefährdet sei, da die auswärtigen Beziehungen der Schweiz, insbesondere zu den USA und Grossbritannien, bei einer Aufnahme Edward Snowdens nachhaltig gestört werden könnten. In dieser Hinsicht müssten jedoch besondere Argumente vorgebracht werden, über die sich derzeit nur spekulieren lässt.

Im Ergebnis müsste Edward Snowden wohl Asyl gewährt werden, zumindest aber müsste er nach Art. 44 AsylG i.V.m. Art. 83 AuG vorläufig aufgenommen werden (als Flüchtling oder als Ausländer ${ }^{46}$ ). Die Rechtsstellung vorläufig aufgenommener Personen ist allerdings nicht mit der von Personen zu vergleichen, die Asyl erhalten haben. Sie gewährt auch - anders als das EU-Recht - keinen besonderen Rechtsstatus, sondern zeugt lediglich davon, dass der Vollzug der Weg- oder Ausweisung (derzeit) nicht möglich ist. Es wird periodisch geprüft, ob die Voraussetzungen für die vorläufige Aufnahme noch gegeben sind (Art. 84 $\mathrm{AuG})$.

39 Voraussetzung für ein Verfahren wäre schliesslich immer, dass sich Snowden in die Schweiz begibt, da eine Asylgesuchstellung an einer ausländischen Botschaft seit Ende 2012 nicht mehr möglich ist.

\section{Fazit}

40 Die angestellten Überlegungen haben gezeigt, dass es durchaus Anhaltspunkte dafür gibt, dass Edward Snowden die Kriterien der Flüchtlingseigenschaft nach der Genfer Flüchtlingskonvention erfüllt und er daher Asyl erhalten sollte. In jedem Fall aber müsste ihm - sofern die Gefahr besteht, dass er unter ähnlichen Bedingungen wie Chelsea Manning inhaftiert würde - aus menschenrechtlichen Aspekten (Grundsatz des Non-Refoulement, insbesondere auf der Grundlage von Art. 3 EMRK) Schutz gewährt werden. In der EU steht hierfür das Instrument des subsidiären Schutzes nach der Qualifikationsrichtlinie zur Verfügung. In der Schweiz müsste Snowden, sofern ihm kein Asyl gewährt würde, jedenfalls vorläufig aufgenommen werden.
45 Ruedi Illes, Art. 83 AuG, Rz. 15, in : Caroni / Gächter / Thurnherr, Bundesgesetz über die Ausländerinnen und Ausländer (AuG), Bern 2010.

46 Der Status vorläufig aufgenommener Flüchtlinge ist im Vergleich zu vorläufig aufgenommenen Ausländern privilegiert, vgl. Ruedi Illes, Art. 83 AuG, Rz. 59, in: Caroni / Gächter / Thurnherr, Bundesgesetz über die Ausländerinnen und Ausländer (AuG), Bern 2010. 